\title{
EDITORIAL
}

\section{Las redes eléctricas del futuro}

En los últimos años, grandes esfuerzos se han realizado para incentivar la utilización de nuevas fuentes de energía en la generación de electricidad, y con ello disminuir el uso de combustibles fósiles en la búsqueda de la sustentabilidad y preservación del medio ambiente. Estos incentivos han generado un nuevo escenario para las redes eléctricas, donde los conceptos de flexibilidad, resiliencia y redes inteligentes (Smart Grids) adquieren gran importancia ${ }^{1}$, frente a las necesidades permanentes de calidad y estabilidad. Por una parte, se requiere de flexibilidad para la incorporación de nuevas fuentes de energía, nuevos tipos de consumos y para permitir la operación de forma híbrida interconectando redes de diferentes frecuencias, niveles de tensión, de corriente alterna o de corriente continua. Por otra parte, se necesita resiliencia para mantener la operación del sistema, o su recuperación, frente a grandes fenómenos naturales que atenten contra su infraestructura y la continuidad de suministro.

Tradicionalmente, los sistemas eléctricos funcionan clasificando a sus componentes en tres sectores según el rol que cumplen: Generación, Transmisión y Distribución. El primero transforma la energía desde una fuente primaria a energía eléctrica. El segundo, Transmisión, provee la infraestructura y los servicios auxiliares para transportar la energía eléctrica a los centros de consumo. El tercero, Distribución, entrega la energía a niveles de baja y media tensión en centros industriales, urbanos o domiciliarios. Este último sector permite el flujo de potencia en un solo sentido, desde la subestación hacia los consumidores, no existiendo la posibilidad de implementar flexibilidad, flujos de potencia bidireccionales o estrategias que permitan la formación de microrredes u operación aislada en modo isla. Junto a este paradigma se encuentra el uso de dispositivos tradicionales para mantener la estabilidad y la calidad del servicio, entre ellos, transformadores con cambio de derivación, bancos de condensadores y reactores, dispositivos con un alto grado de confiabilidad y eficiencia, pero con una baja capacidad de control y flexibilidad, no permitiendo el uso de estrategias actuales de control, el uso del avance tecnológico en nuevas topologías y dispositivos de electrónica de potencia.

De todas formas, algunos avances tecnológicos si han logrado impactar en el sector de transmisión, al incluir el uso de Sistemas Flexibles de transmisión de Corriente Alterna ${ }^{2}$ (FACTS, por su sigla en inglés) o la transmisión de energía eléctrica en corriente continua de alta tensión, permitiendo flexibilidad al controlar los flujos de potencia y otras características dinámicas del sistema. Sin embargo, aún es posible realizar un paso más allá respecto a la flexibilización en la operación de las redes eléctricas, sin perjudicar la estabilidad y la resiliencia del sistema, particularmente aplicables al sector de Distribución.

En la actualidad, el desarrollo en áreas como la Electrónica de Potencia, Control Automático, Computación, Comunicaciones, Almacenamiento de la Energía, entre otras, ha permitido ampliar la cantidad de participantes en nuestras redes eléctricas, considerando como necesidad clave la integración de Medios de Generación Distribuida, Microrredes, Vehículos Eléctricos y Prosumidores ${ }^{3}$, todos

1 B.K. Bose, "Power Electronics, Smart Grid and Renewable Energy Systems", Proceedings of the IEEE, vol. 105, No 11, pp. 2011-2018, 2017, doi: 10.1109/JPROC.2017.2745621.

2 F.Z. Peng, "Flexible AC Transmission Systems (FACTS) and Resilient AC Distribution Systems (RACDS) in Smart Grid", Proceedings of the IEEE, vol. 105, No 11, pp. 2099-2115, 2017, doi: 10.1109/JPROC.2017.2714022.

3 C. Roncero-Clemente, N. Vilhena, V. Delgado-Gomes, E. Romero-Cadaval and J.F. Martins, "Control and operation of a threephase local energy router for prosumers in a smart community”, IET Renewable Power Generation, vol. 14, No 4, pp. 560-570. [Online]. Available: https://digital-library.theiet.org/content/journals/10.1049/iet-rpg.2019.0589 
ellos habilitados por convertidores de potencia, lo cuales permiten descentralizar la Generación de electricidad, pero por otro lado, creando el desafío de pensar en cómo las redes eléctricas funcionarán en las próximas décadas.

Las características preliminares que las redes eléctricas podrían requerir en el futuro son: un sector de Distribución flexible, resiliente, estable y confiable, que permita la interconexión de nuevos actores a distinto nivel y de forma distribuida, y con flujos de potencia bidireccionales; un sector de Transmisión también flexible, que permita la integración de nuevos medios de generación, en corriente continua o alterna, y almacenamiento energético a gran escala; finalmente, un sector de Generación que disminuya el uso de combustibles fósiles. Con lo anterior, es posible prever que la exclusividad en la operación de cada sector en el sistema eléctrico necesariamente tendrá que abrirse a incluir nuevos participantes, tanto desde un punto de vista económico y como técnico. Un primer paso en esa dirección es la implementación de la Generación Distribuida, que consiste en la conexión de sistemas de generación a la red de distribución. También el fomento a la instalación de sistemas de generación domiciliarios ha sido un gran aporte. De todas formas, continúan apareciendo nuevos desafíos como la conexión de vehículos eléctricos (tanto como carga o como generadores), así como también las nuevas topologías para redes de baja potencia como son las microrredes.

Hoy en día, y principalmente en el entorno académico, uno de los ámbitos de estudio que mayor atención está obteniendo, en referencia a las redes eléctricas del futuro, es precisamente el cómo lograr integrar todos estos elementos para mantener una operación estable, resiliente, confiable y de calidad, considerando la integración de distintos medios, con diferentes frecuencias en el caso de corriente alterna o de corriente continua. Lo anterior solo es posible con el uso de convertidores de estado sólido. Se espera que los nuevos dispositivos basados en carburo de silicio ( $\mathrm{SiC}$, por su sigla en inglés), que permiten operar en un mayor rango de frecuencia y a mayores niveles de tensión, apoyen el desarrollo y diseño de conversores para esta tarea. Además, el avance en la generación de nuevas topologías de conexión para convertidores, como son los Conversores Multinivel, las nuevas estrategias de control y el avance en capacidad de cómputo, permitan a su vez hacer de estos sistemas más confiables y, con el tiempo, más económicos. Estas últimas dos características (confiabilidad y costo) son precisamente las únicas ventajas que los dispositivos tradicionales mantienen, pero que el futuro ayudará a superar.

Existe una propuesta muy interesante de arquitectura para las redes eléctricas del futuro a nivel de Distribución, y se trata de concebirla de forma similar a una red de internet. En esta conceptualización la conexión de prosumidores (microrredes, vehículos eléctricos, medios de generación distribuida, etc.) a la red eléctrica sería tan simple como la conexión de un equipo computacional a internet. En este concepto, las actuales subestaciones de distribución operarían como enrutadores de red, permitiendo al interconexión y administración inteligente de los flujos de potencia. Esta proyección hacia el futuro recibe el nombre de Energy Internet ${ }^{4}$, y el reemplazo para las subestaciones reciben el nombre de Energy Router, Intelligent Power Transformer, Smart Transformer, Power Router, entre otros ${ }^{5}$. La clave para su implementación se encuentra en el estudio de los transformadores de estado sólido ${ }^{6}$ (SST, por sus siglas en inglés) y en cómo integrar en ellos las características de los nuevos dispositivos de

4 A. Joseph and P. Balachandra, "Smart Grid to Energy Internet: A Systematic Review of Transitioning Electricity Systems", IEEE Access, vol. 8, pp. 215787-215805, 2020, doi: 10.1109/ACCESS.2020.3041031.

5 Y. Ma, H. Liu, X. Zhou and Z. Gao, "An Overview on Energy Router Toward Energy Internet", in 2018 IEEE International Conference on Mechatronics and Automation (ICMA), 5-8 Aug. 2018 2018, pp. 259-263, doi: 10.1109/ICMA.2018.8484645. [Online]. Available: https://ieeexplore.iee.org/document/8484645/

6 M.A. Shamshuddin, F. Rojas, R. Cardenas, J. Pereda, M. Diaz, and R. Kennel, "Solid State Transformers: Concepts, Classification, and Control" Energies, vol. 13, No 9, p. 2319, 2020. [Online]. Available: https://www.mdpi.com/1996-1073/13/9/2319. 
electrónica de potencia, control, computación y ciberseguridad, de tal forma que su operación, además de eficiente y confiable, sea inteligente.

Todo lo anterior será posible en el futuro, siempre que se logren mayores avances en la academia, popularidad en el sector industrial y, por sobre todo, mayor conciencia sobre la sustentabilidad en el uso de nuevos medios de generación de energía eléctrica, partiendo desde los usuarios domiciliarios, pasando por las industrias, hasta los sectores de generación, transmisión y distribución, es decir, no solo convertirnos en parte activa de la red eléctrica generando o consumiendo energía, si no también, convertirnos en parte activa del ciclo que considera la transformación de la naturaleza en energía eléctrica.

Pablo Guicharrousse

Universidad de Tarapacá

Departamento de Ingeniería y Tecnologías Iquique, Chile

E-mail: pguichar@academicos.uta.cl https://orcid.org/0000-0003-0116-5555 